\title{
Report of an IEEE Task Force-An IEEE Opinion on Research Needs for Biomedical Engineering Systems
}

\author{
ALFRED R. POTVIN, SENIOR MEMBER, IEEE, WILLIAM G. CROSIER, MEMBER, IEEE, ELI FROMM, \\ FELLOW, IEEE, JAMES C. LIN, SENIOR MEMBER, IEEE, MICHAEL R. NEUMAN, MEMBER, IEEE, \\ THEO C. PILKINGTON, FELLOW, IEEE, CHARLES J. ROBINSON, SENIOR MEMBER, IEEE, \\ LAWRENCE W. SCHNEIDER, JOHN W. STROHBEHN, SENIOR MEMBER, IEEE, PETER SZOLOVITS, \\ AND WILLIS J. TOMPKINS, SENIOR MEMBER, IEEE
}

\section{INTRODUCTION}

$\mathbf{M}^{\circ}$ ORE than 200 years ago, our forefathers made note of man's inalienable rights to life, liberty, and the pursuit of happiness. To the engineering community (applied science in the service of man), these may be coincident with applications to medicine and biology (biomedical engineering), defense, and entertainment. Biomedical engineering research has the distinction, among these three missions, of not only contributing to the quality of human life through the industrial economy but also to life itself - the most fundamental concern of all people. It is through biomedical engineering research that we have been able to learn much concerning the functioning of living systems, and it is through such knowledge that we have been able to develop improved clinical diagnosis, monitoring, and treatment, including life-sustaining devices

Manuscript received May 8, 1985. This paper was prepared by an EMBS committee on behalf of the IEEE and submitted on request to the National Research Council in March 1985. The NRL established the Engineering Research Board to take an overview of current engineering research programs and to identify areas for future research. This report was one of five submitted by the IEEE. Requests for reprints should be addressed to A. $R$. Potvin, Director, Medical Instrument Systems Research Division, Eli Lilly and Co., Lilly Corporate Center, Indianapolis, IN 46285.

A. R. Potvin is with Eli Lilly and Company, Medical Instrument Systems Research Division, Lilly Corporate Center, Indianapolis, IN 46285.

W. G. Crosier is with the Biomedical Research Laboratories, Technology, Inc., Houston, TX 77004.

E. Fromm is with the Department of Electrical and Computer Engineering and the Biomedical Engineering and Science Institute, Drexel University, Philadelphia, PA 19104.

J. C. Lin is with the Robotics and Automation Laboratory, College of Engineering, University of Illinois, Chicago, IL 60616.

M. R. Neuman is with the Department of Reproductive Biology, Case Western Reserve University, Cleveland, $\mathrm{OH} 44106$.

T. C. Pilkington is with the Department of Biomedical Engineering, Duke University, Durham, NC 27706.

C. J. Robinson is with the Rehabilitation R\&D Center, Veterans Administration Edward Hines, Jr., Hospital, Hines, IL 60141.

L. W. Schneider is with the University of Michigan, Ann Arbor, MI 48109.

J. W. Strohbehn is with the Thayer School of Engineering, Dartmouth College, Hanover, NH 03755.

P. Szolovits is with the Department of Computer Science and Engineering and the Clinical Decision Making Group, Massachusetts Institute of Technology, Cambridge, MA 02139.

W. J. Tompkins is with the Department of Electrical and Computer Engineering, University of Wisconsin-Madison, Madison, WI 53706.

IEEE Log Number 8406107. and aids to the handicapped. Each step represents an improvement in the quality of life, and each step forms the foundation upon which to gain new knowledge to improve upon earlier developments.

Biomedical engineering research has been at the systems level (simulation, control mechanisms, imaging, signal analysis and interpretation, fluid dynamics, mechanics, assistive devices, health management, etc.) as well as the cellular level (interaction of ultrasonic, electric, and electromagnetic energy with biologic tissue). The future portends a similar mix from the application of concepts of robotics (control, mechanics, computer and information systems, and machine vision) to closed-loop control of drug delivery, and closed-loop control of artificial organs and prosthetics-developments which will lead to fully realized substitute life functions and the molecular/cellular/ systems work of biosensors. Such innovations and advances in the state of the art of the products of the U.S. biomedical engineering industry will support the health of the U.S. industrial economy as well as directly enhance the quality of life of the people.

\section{Research Priorities}

In this section, we describe the eight areas that we have identified as having high-priority needs for research funding:

- biosensors

- medical imaging

- cardiac assist devices

- medical artificial intelligence and information systems

- medical robotics and automation

- rehabilitation engineering

- mechanisms of injury and protection in accidents

- artificial environments.

Topics that have been omitted should not be construed as unimportant. Instead, the topics included are those that the panel considered most important.

\section{A. Biosensors}

Biosensors transduce biologic information into an electronic signal that can be processed by an instrumentation 
system. The availability of sophisticated signal processing hardware and software, and actuators to control therapeutic devices, make the need for improved biosensors acutesensors are oftentimes the weakest link in an instrumentation system. Improved biosensors and instrumentation systems can significantly affect the health of our population through earlier detection of pathology. Early detection can result in more effective, lower cost treatment; provide better, more reliable data during critical care situations; allow more medical care to be accomplished outside traditional hospitals and clinics; and assist our aging population by making it possible to monitor individuals at home rather than in institutions. Improvements in biosensors could make individuals more productive by minimizing time away from work due to illness; reduce health care costs by permitting more health care and convalescence to take place in the home rather than in the hospital; provide clinicians with more objective data to aid clinical decision making; and lead to prosthetic devices to help patients regain physiologic functions lost as a result of disease or injury.

Future biosensor research could help scientists better understand the body's natural sensors and actuators. Research could result in obtaining signals from the body's natural sensors and interfacing a device to the nervous system. Smaller, more reliable and more reproducible sensors could be fabricated by mass production using micromachining technology from the microelectronics industry. Such sensors could be directly integrated with signal processors or preprocessors, in a redundant way to improve reliability. With self-test algorithms incorporated into the sensing system, unreliable sensors could be ignored. These possibilities require application of a broad systems approach to development of sensors and instrumentation systems. Such an approach should include not only signal processing aspects, but also biocompatibility, placement, lifetime, and interface to the body.

An important problem in sensor design that needs to be addressed is the compatibility of biosensors with signal processing systems. Today; there have been great advances in digital signal processing, but for the most part, existing sensors provide analog signals as their output. The analog signals must be converted to digital form either at the sensor or at the signal processor. The nervous system is an example of a system which utilizes sensors that put out a digital-type signal. Artificial sensors with digital outputs that could interface directly with digital systems should be developed.

Sensors can make an important contribution to the health of our citizens. Today, we are close to being able to provide artificial closed-loop control systems to replace failed physiologic functions of the body. Such control systems should sense the physiologic variables they control.

Noninvasive or minimally invasive sensors should be developed for diagnostic and therapeutic monitoring so as to permit the widest possible use. With success, more routine diagnostic tests could be carried out on a larger population with paraprofessionals performing the procedures.
Possibly, diagnostic and therapeutic monitoring could be carried out by an individual at home using such instrumentation. The health care provider could obtain more representative and more reliable data than those obtained in a health care institution.

Home health care products are now emphasized as a means of reducing health care costs and improving the care of patients confined to the home. The key to a successful home-monitoring system involves biosensors and microcomputer-based signal processing. Small computer systems that have the advantages of mass production (i.e., low cost, high reliability, and maintainability) could be interfaced to various biosensors so that it would be possible in the patient's home to acquire physiologic data such as blood pressure, heart rate, body temperature, respiratory rate, oxygen and carbon dioxide content of blood, and $\mathrm{pH}$ of blood. The system could be "intelligent" enough to detect potentially dangerous clinical problems. It could then communicate with a hospital computer and send collected data over the voice-grade telephone system automatically. A central computer system could be designed that would receive the data from multiple remote sites and maintain a database on each patient.

Research should support the transfer of some of the health care technology now available only in hospitals to the home environment [1]. However, the design of such a system would have to be quite different from a hospital system since the home would be a remote sensing environment with health care workers only distantly available. The design should necessarily emphasize reliability, simplicity of use, and redundancy.

An important emerging area of biosensors involves chemical sensors for both clinical laboratory and in vivo monitoring. Bedside monitoring of blood and urine chemistry would provide the clinician with results immediately rather than $15 \mathrm{~min}-2.5 \mathrm{~h}$ after examining the patient, permitting improved treatment, earlier discharge, and lower medical costs.

Even areas that have received much clinical attention, such as cardiac research, still have less than optimally accurate, reliable, and practical measures of such parameters as blood pressure and cardiac output. These quantities are crucial in the care of patients, and noninvasive or minimally invasive methods for obtaining these variables should be developed.

The biosensors area is one for fruitful and exciting potential combination of multiple disciplines including electrical engineering, materials, membranes (biologic and synthetic), solid-state electronics and physics, mechanical engineering, chemical engineering, and biochemistry. Research in areas such as membranes, allowing controlled charge transfer, adherence to semiconductor surfaces, coupling of biologic molecules to traditional electronic and electrooptic circuits, will offer exciting future possibilities for control of therapeutic devices, closed-loop pharmaceutical administration, artificial organs, and prosthetics. It is a difficult, challenging research area having tremendous implications for improved health care. 


\section{B. Medical Imaging}

Medical imaging has been one of the fastest developing areas of medical technology during the last 15-20 years. The invention of X-ray CT (computed tomography) by Nobel Laureate Godfrey Hounsfield in the late 1960's has led to a revolution in medical imaging that had not been matched since the creation of the field of medical imaging based on the original discovery of X-rays by Roentgen in 1895. However, a whole host of other new imaging technologies has also been under development during this period, including magnetic resonance imaging (MRI, also called NMR or nuclear magnetic resonance imaging), ultrasonic imaging, digital radiography, emission computed tomography, and nuclear imaging. These advances, almost all of which have been intimately tied to the development of powerful small computers, are revolutionizing the practice of modern medicine. For example, the introduction of $\mathrm{CT}$, an outpatient procedure, has almost eliminated the use of pneumoencephalography, an expensive and painful procedure requiring hospitalization. The benefit to the quality of life of these new technologies needs no elaboration.

The actual development of the CT scanner was foreshadowed by a series of fundamental studies in such diverse fields as mathematics, radioastronomy, and electron microscopy dating back as far as 1917 , although most of the relevant work appeared in the late 1950's and 1960's. Much of the early work was carried out in academic settings, but the work of Hounsfield was performed in the Central Research Laboratories of Electro-Musical Instruments, LTD. This history is similar to that in many of the other medical fields, i.e., fundamental ideas are often discovered in the university setting but the actual realization and development are often done by industry.

While much progress has been made as a result of the CT scanner, more remains to be done. Research should be aimed at developing three-dimensional images and at generating X-ray holograms that could be examined from several viewpoints.

At the present time, the imaging modality receiving major attention both by the medical community and by industry is magnetic resonance imaging (MRI). Fundamentally, X-ray CT maps the specific gravity of the brain and reveals its morphological structures. However, it is not able to characterize the tissues or to give information about organ chemistry and function. The tremendous interest in MRI is based on its potential for providing the latter type of information in the form of high-resolution images that can be created at any angle or location. MRI is based on the interaction of a selected atom, an external magnetic field, and an external radio-frequency electromagnetic field. In medical imaging, the selected atom has usually been the proton, thus, the physician is able to visualize the proton distribution in a particular plane of the patient. With adequate research support, MRI will eventually create as great a revolution in medical imaging as the $\mathrm{CT}$ scanner because of its potential capability to provide data on the patient's biochemistry and pathophysiology.

The CT scanner and MRI, as well as ultrasonic imaging and emission tomography, have all made significant advances in recent years. Future work should be directed toward combining imaging and function measurements, as is being explored with pulsed Doppler devices. A successful system could once again revolutionize the practice of diagnosis and monitoring.

Over the past two decades, rapid developments in the imaging field have been predicated on fundamental discoveries in academic and industrial settings coupled with aggressive industrial development of the technology. Recent changes in both regulation and financing of medical care and research, such as regulations promulgated by the FDA, third-party payment policies, and certificate-of-need programs, could conceivably affect this type of growth in the future. Presently, there is a significant worldwide competition in the medical imaging field, and any factors that impede U.S. research and development activities will affect this competition. Accordingly, research support for medical imaging is perhaps even more important in the future than it has been in the past.

\section{Cardiac Assist Devices}

One million Americans die of cardiovascular disease each year, and tens of millions are chronically disabled. Americans spend over $\$ 2$ billion annually on coronary artery surgery, and the cost of the disease in terms of lost productivity is probably an order of magnitude greater than the cost of surgery. Thus, less traumatic, more effective treatments for coronary artery disease would be a major contribution to public health-and to the economy.

Modern implantable pacemakers have evolved from fixed-rate pacemaker designs into sophisticated arrhythmia detectors [2] that directly benefit about 500,000 persons in the U.S.A. today and twice this number worldwide. Implantable defibrillators, cardioversion systems, and exercise-responsive pacemakers using state-of-the-art microelectronics appear promising [3]. However, cardiac physiology suggests strongly that these devices and systems have not yet achieved their full potential. Their design is based almost completely on temporal data while, in fact, the bioelectric activity of the heart is highly spatially dependent [4]. Viable quantitative descriptions of the mechanisms of bioelectric alteration, both spatial and temporal, could provide the formulations and underpinnings essential for the design of electrode configurations that maximize cardiac performance and minimize risk.

Fortunately, biomedical engineers in the last decade have begun to explore, develop, and evaluate methodologies for quantitatively relating electric field and physiologically accurate bioelectric sources. Finite element and integral equation models have been used to relate cardiac generators to electric field distributions, and the agreement between simulations and experimental measurements is im- 
pressive [5]. This work, combined with recent developments in basic cardiovascular research in structure and function, should lead to an accurate probabilistic description of the implications of possible pacing or stimulation modalities. Much, of course, remains to be done. This base of biomedical engineering knowledge should be combined with state-of-the-art microelectronics to develop implantable cardiac interventional systems (pacemaker, defibrillator, or cardioversion) that may provide the optimal support modality for assessing, intervening, and sustaining cardiac performance.

A reasonable estimate of the market potential of cardiac pacing and antiarrhythmia systems is that the industry could double in the next 6-8 years, growing from 1 to 2 billion dollars per year.

Several proposed interventional techniques utilizing vascular endoscopy hold considerable promise pending advances in widely divergent areas. The ability to guide catheters into the heart and coronary arteries to visualize and manipulate tissues there has already alleviated some of the need for open-chest surgery. It is anticipated that the advancement of laser and ultrasound energy delivery systems and microsurgical tools compatible with these catheters could further obviate major surgical procedures such as coronary bypass for removal of obstructive atherosclerotic plaque.

Current systems are limited by the efficiency and flexibility of fiber optic materials, image quality of catheter endoscopes, and the utility and ease of use of microsurgical tools. In addition, further basic research on the interaction of laser and ultrasonic energy with thrombus and atherosclerotic plaque is required [6].

Laser angioplasty is now of great interest [7]. The major problem is the removal of the desired material, the plaque, without puncturing the vessel wall. One possible procedure is to enlarge the lumen sufficiently by using an Nd-argon or similar laser which can operate through a saline- or air-filled transparent balloon presséd against the material to be removed. Another possibility is to use an air or carbon dioxide bubble interfacing with the tissue to allow a $\mathrm{CO}_{2}$ laser to be used, but a mid-range infrared laser (near $2.9 \mu \mathrm{m}$ ) may be better since it can ablate the wall in very small steps. There are many variations of this approach, and most are extremely fruitful avenues of investigation to follow. Much more research is needed to show the factors involved in this type of laser-tissue interaction. The market potential of laser angioplasty products alone has been estimated at $\$ 400$ million per year.

\section{Medical Artificial Intelligence and Information Systems}

The art and science of medicine has already combined the health practitioner's reasoning abilities with the use of a growing armamentarium of diagnostic and therapeutic tools to provide more effective, safer, and perhaps more efficient health care. The field of medical information sci- ence (recently also called "medical informatics") focuses on augmenting the reasoning ability of the physician by providing improved access to data, medical knowledge, and-through the use of recently developed artificial intelligence (AI) techniques-case-specific advice in the form of consultation, error monitoring, and explanation programs.

The two key interrelated areas of research for advancing the state of this art are 1) formalization and codification of medical knowledge [8], and 2) development and application of artificial intelligence techniques for understanding, simulating, and enhancing clinical reasoning [9], [10].

All medical knowledge, from the world's medical literature to a physician's notes on the care of an individual patient, is recorded in natural language (e.g., English). The richness of expression allowed in ordinary language is a benefit to the writer, but makes it difficult for any computer program to categorize, organize, sift, retrieve, or reason with this knowledge. Indeed, most current retrieval systems deal with the text of documents and patient records rather than the content, and only very limited successes have been achieved with early rigid coding schemes. The study of how real-world knowledge is to be encoded in the computer to support the use of its content is called knowledge representation. Research in this field is critical to form the basis for new methods of formalizing and codifying medical knowledge, both for human and machine consumption.

The advantages of formal schemes of medical knowledge representation are many. Advances here should support improved bibliographic retrieval systems, thus making the utilization of hard-earned knowledge more effective; they should provide the basis for medical recordkeeping systems that capture essentially the total medical record, laying the base for new hospital information systems, more systematic epidemiological studies, and medical consultation systems (see below).

In addition to research on knowledge representation, a number of systems issues must be addressed. How are new knowledge bases to be collected, how are they to be validated, and how are they to be disseminated? New knowledge can be captured from experimental data, abstracted clincial experience, or the judgment of human experts. Must this be done manually, or can automated methods of data capture and debriefing be used? Can methods of natural language understanding be applied to "read" at least limited ranges of published material? Knowledge bases can be validated by consensus, by methods adapted from textbook publishing, and by the experimental use of the knowledge in reasoning programs, to explore its consequences. Making the knowledge available requires carefully identifying the information needs of the user community; the key is that retrieval must be possible by reference to factual content, not simply text.

If research projects in codifying medical knowledge are successful, then it will also be necessary to make institutional arrangements to create "certified" bodies of re- 
liable knowledge for use as reference works by medical practitioners and as the underlying knowledge base by computer programs.

Programs based on artificial intelligence (AI) methods reason by simulating the inferences and associations thought to underlie human reasoning abilities. The chief advantage of this approach and the technologies that implement it is that it is innately very flexible, permitting the encoding of new types of knowledge and new heuristics and strategies when they seem to be demanded by the application. Further, because of the similarity between the inferential steps of such a program and human thinking, the conclusions and reasoning methods of the program can be explained to its human users in meaningful terms.

Principal applications of AI programs can be as consultants, error monitors, intelligent instrument interpreters, and teachers. A consultation program can encode the expertise of specialists and make it available to less-expert practitioners, just as a human consultant might; such a program could be much more easily consulted, however, and would be more widely available. In the consultation mode, a program could give diagnostic and therapeutic advice, critique an already-formulated plan of intervention, help think through alternative interpretations of a case, and relate it to knowledge bases extracted from the literature or from other patient histories. In a more automatic mode, similar systems could serve as error monitors, reviewing patient case records in the background and notifying the user when a discrepancy was detected. Such a program would impose almost no additional interaction requirements on the health care system (a great advantage) if it were coupled with an automated hospital information system from which its information about patients could be drawn. As medical laboratory instruments become more sophisticated, another possible application of AI methods is to help interpret a large number of interrelated measurements from such an instrument to suggest the possible clinical relevance of the results. Two such interpretation programs, for pulmonary function and serum electrophoresis measurements, are now in use, and many more could be effective. Education programs could take a variety of forms, from a patient simulation that first permits a student to intervene in a simulated case, then provides a critique of that intervention to very sophisticated programs that can explain the relationship between clinical facts and basic science principles. Principal research directions here are studies of human clinical cognitive processes; effective knowledge representation schemes; useful qualitative models of human anatomy, physiology, disease processes, interventions, etc.; use of simulation in reasoning; incorporation of notions of likelihood and utility in reasoning models; hypothesis generation, testing, and revision; and integration of shallow reasoning based on associations between diseases and their manifestations with "deep" reasoning based on pathophysiology; use of planning methods for developing and evaluating patient management plans; improved methods of explanation and justification; integration of reasoning programs with more traditional med- ical records systems to provide integrated automatic access to patient information for the reasoning program; and integration with validated knowledge bases, to provide its basis for reasoning.

In addition to the above-described frontier research areas, one other funding need is important to identify: support for career development for junior investigators in the interdisciplinary domain combining computer science and medicine, and stable funding for larger long-term research projects. The general prospects for artificial intelligence applications in military, financial, and industrial areas are so hot at the moment that it is difficult to retain well-trained young researchers in the field of medical AI and medical informatics. Young researchers face difficult competition for research funding, compared to much more widely available private and governmental funding for other application areas. In addition, research efforts to realize many of the goals outlined above are time consuming and require a well-coordinated interdisciplinary team. If funding is not reliably available for five-year or longer projects, the effort of assembling and integrating such a team and of competing for follow-on funds can absorb a large fraction of the productive energies of a project.

With suitable funding, the information revolution should extend to medicine, providing an effective codification and collection of medical knowledge and intelligent programs that exploit it to improve medical care.

\section{E. Medical Robotics and Automation}

Research in biomedical robotics and automation is complex and interdisciplinary in nature. It must draw upon the diverse talents of bioengineering researchers to produce significant progress. Nevertheless, it promises to usher in a new technological era in which computers and robots combine to give sight to the blind, movement to the disabled, relief to the infirm, and hope to future generations.

Important areas of biomedical robotics and automation research that need engineering solutions are many, but we believe that increased attention should be directed toward the following areas:

- artificial limbs

- robotic aids

- robotics for the clinical laboratory

- robotics for the operating room.

Advances in artificial limbs for the physically disabled have been impressive in recent years. The microprocessor has rendered heretofore unthinkable problems amenable to technological solutions [11]. Sustained research efforts are required to permit artificial arms and legs to have a wide range of functions and capabilities. This would involve new initiatives in control signal acquisition and processing schemes and in algorithms to transfer input signals to multiple commands with minimal lag time. The prosthetic devices would necessarily be designed for reliability and ease of use and field repair.

Research in robotic aids for handicapped and geriatric 
populations represents another area requiring attention. The use of robotics may enable these individuals to perform such basic tasks of daily living as working, housekeeping, communication, entertainment, and companionship.

Although in an early stage of development, the integration of computer-controlled robots into the clinical laboratory offers advantages of performing prescribed tests and assays more accurately, efficiently, quickly, and reliably. This integration will free skilled technologists from tedious, repetitive, and well-defined tasks toward tasks more appropriate for their training. Such integrated systems combine data acquisition, analysis, and display, and provide a permanent record for accurate documentation [12], [13]. In addition to special purpose manipulators and end effectors, this system would require further development in control languages, interface protocols, and bench instruments that conform to automation. Moreover, significant improvements in test performance could be achieved by suitably changing the laboratory environment to the robot's special capabilities.

Introduction of robotics into the hospital operating room is attractive in that it holds the promise of reduced patient risk, enhanced surgical efficacy, and increased health care efficiency. Utilizing anatomic data generated by modern imaging modalities as the knowledge base, the motions of a robot manipulator could be coordinated to effect certain surgical procedures, perform delicate biopsies, position implant devices, or deposit therapeutic agents inside the body with accuracy and precision difficult to match by human hands [14]. While this technique could permit $a$ priori determination of manipulator trajectory and obviate much of the need for direct visualization, integration of tactile sensing would provide feedback to avoid complications arising from unexpected obstacles during surgery. An adjunct to this development would be the robot's ability to respond to verbal commands from human operators. In addition, speech recognition would be an important training mode for automated information retrieval systems that could provide immediate access to crucial medical history and pertinent surgical information in the operating room, allowing the surgeon to respond to changing conditions.

The goals of medical robotics and automation are in reach, the research will improve health care and quality of life, and the research breakthroughs have applications far beyond the medical field.

\section{F. Rehabilitation Engineering}

The term rehabilitation engineering has been applied to the multifaceted research and development activities directed toward bettering the daily lives of the physically and sensory disabled.

Tantalizing glimpses of what is currently possible through the application of technology to the needs of the disabled include voice-operated robotic arms and tables, electric activation of paralyzed limbs, use of space-age materials and concepts in wheelchair and prosthetics design, cochlear implants, and voice synthesizers [15]. Un- fortunately, many of the fruits of rehabiliation, while often dazzling, have not been translated into affordable commercial products acceptable to the disabled end user. On the one hand, this lack of technology transfer points out the need for increased research in certain areas of the rehabilitation field; on the other hand, it also indicates that a restructuring is probably warranted in the traditional roles played by academia, government, and industry in the research and development of devices for the disabled consumer [16], [17].

The marriage of physiology, electronics, and systems engineering could have great potential in one emerging area in the rehabilitation field if some fundamental engineering problems could be solved. The lack or loss of sensation and/or control of movement due to congenital or traumatic insult to the central nervous system often results in a less-than-satisfactory quality of life for the individual affected. We can now move paralyzed limbs (but without precise control); provide the deaf with auditory sensations (albeit crude) and the vocally impaired with mechanically sounding speech aids; and provide partial recovery to stroke patients through traditional rehabilitation methods (which for the most part depend on the skill and artistry of the clinicians involved). A coordinated engineering research effort focused on all aspects of the motor sensory rehabilitation problem during the next decade could yield impressive breakthroughs.

From a systems standpoint, we need to know more about the control strategies used in normal movement and speech, how these strategies are influenced by various lesions within the central nervous system, and how we might use an engineering analysis of these modified strategies to guide retraining after neural insult in cases where functional recovery might be possible. Barring complete recovery, are there ways that we might be able to augment whatever residual function remains by somehow sensing "intent" and supplying artificial activation or assistance? Two examples are the resynthesis of poorly articulated speech and the control of hand function in quadriplegics by sensing shoulder position. While these examples are disparate, they have common themes that can be used to point out some other aspects where complex engineering problems remain.

Acceptable solutions will require advances in pattern recognition (i.e., the unique signal that indicates what I want to do); knowledge of state (where in the process of moving or speaking am I?); precise control (how do I get to the next state in a natural manner?); physiologically appropriate activation or substitution (can I use existing muscles or must I have a substitute?); and in the understanding of the unique "human factors" considerations that must be made ("my design is an engineering masterpiece! - why won't the handicapped use it?").

Some of these advances will need to come in the development of miniature artificial sensors and actuators that can monitor limb position, pressure, vibration, effort, etc.; reliably decode signals directly from peripheral nerves or central nervous system structures; or activate such struc- 
tures. With such devices, closed-loop control would become possible, provided that we knew what control strategy to use in what situation. For limb control, these devices will most probably need to be implanted. Although heart pacemaker development has been an exceptional example of such implanted technology, the sensing and control engineering needed to move paralyzed limbs are many orders of magnitude more complex. And although such devices might be practical, no one yet knows how many disabled individuals will ultimately want to subject themselves to such "bionic" implants!

Considerations of the latter type have most often been ignored in current rehabilitation research and development activities [16], [17]. We really need an extensive analysis of the factors that the disabled consider important in products made for their use and how they would actually control such devices. Without such an analysis, advancements in the field of rehabilitation technology will remain impeded. The problem of interfacing people and equipment in an integrated way, so that function is maximized and the mental load needed is minimized, is a difficult one. Does a particular product represent an optimal balance between the needs and abilities of each disabled individual and the appropriateness of the technology used? For instance, why should a vocally impaired individual be forced by product design to have synthesized speech that sounds computer generated? Should that individual not be able to select a voice appropriate for gender, age, dialect, and familial pattern? With enhancements in speech algorithms and computer miniaturization over the next decade, the cost of such natural and customized speech could be brought into an affordable range.

The last point to be made concerns the traditional roles played in engineering research by academia, government, and industry. The initial impetus for rehabilitation product design can arise from well-intentioned clinicians, paraprofessionals, engineering faculty and students, or staff in government and industrial labs, or from the disabled themselves. Products designed specifically for disabled consumers almost always have a low market potential, require limited manufacturing runs, and may need custom fitting. Often, only a prototype exists. Hence, the price of such products must be high for a manufacturer to make an acceptable profit and cover potential product liability. Many inexpensive products on the general commercial market could have been used by disabled consumers if minor changes could have been made early in the design process. Product developers need to become sensitive to such possibilities. There should be economic incentives for a manufacturer to produce these orphan products in terms of tax relief, liability assumption, or patent protection. A strong argument can be made for devices that have been developed to improve an individual's productivity and reduce the cost burden of government assistance. The potential economic benefits to society are considerable.

The future appears to be most promising for the disabled. With adequate research funding and appropriate guidance, rehabilitation engineering holds forth the promise of a better quality of life for disabled individuals.

\section{G. Mechanisms of Injury and Protection in Accidents}

Accident-induced injury to the human body is the fourth leading cause of death in the United States, exceeded only by heart disease, cancer, and stroke. For persons in the age range between 1 and 44 years, it is the leading cause of death; for persons between 5 and 44, accidental trauma kills more people than all other causes combined [18]. Trauma to the central nervous system is the most frequent cause of accidental death and permanent disability (i.e., paraplegia, quadriplegia, brain damage). Of all accidental deaths, motor vehicle accidents account for more than all other accident sources combined. It has been estimated that injuries due to motor vehicle crashes in 1975 cost nearly $\$ 15$ billion, second only to the costs associated with cancer [19]. Over half of these costs are associated with central nervous system injuries to the head and neck.

The statistics of accidental deaths and injuries are indeed impressive and so are the economics. Because the rates of disabling injuries and death are highest among the young, the costs and losses to society are great.

The prevention and minimization of accidental deaths and injuries is a public health challenge that, to a great degree, is in the hands of engineers. The rational design of protective devices and systems requires an understanding of the mechanical processes that lead to injury, as well as knowledge and definition of the tolerance levels of human tissues and organs to impact forces and accelerations. Yet, current knowledge of the biomechanics of trauma and injury tolerance is far from complete.

During the past ten years, experimental techniques for studying impact trauma have benefitted greatly from advances in electronic measurement and digital data processing technology. Computer technology has also provided the opportunity for significant developments in analytical methods used to simulate the human response to external forces and accelerations through mathematical models. Unfortunately, while technology has moved forward in this period, the research funding necessary to allow broad-based, comprehensive biomechanical studies of trauma has shrunk.

As a consequence, few research groups currently specialize in the biomechanics of trauma, particularly in the university setting, and those that do are often limited by inadequate funding for necessary staff and research equipment. In comparison to other medical problems of similar magnitude, funds allocated to basic studies of trauma are very limited and new researchers are not being attracted to the field. There is currently a great need to support university programs and graduate research in biomechanics of trauma to rectify this situation.

Carefully planned and well-funded research programs that coordinate the results of biomechanical studies and impact studies using animals, human cadavers, and living subjects with data from accident investigations and the re- 
sults of mathematical computer simulations are required to get the needed information efficiently and quickly [20]. Particular focus and emphasis must be given to central nervous system and soft tissue injuries of the head and neck and to a better understanding of the roles of linear and angular accelerations in producing injury to these tissues. Research funds are also needed to:

- collect better and more complete anthropometric data of humans including dimensions, mass, shape, and inertial properties;

- define the mechanical properties of human tissue and the dynamic failure modes and tolerance levels of tissues and organs;

- augment the application of mathematical models to the problems of understanding biomechanic response and tolerance to impact, and to extend the development of these models to include the nonlinearities associated with large strains of human tissues; and

- study the biomechanics and biodynamics of human joints to enable better definition of joint mobility, joint response to inertial loading, and the role of active (i.e., muscle) and passive (e.g., ligament) tissue in impact response.

Trauma research aimed at defining human tolerance to injury and understanding the mechanisms of injury is difficult and expensive, but the costs of not doing this research are significantly greater.

\section{H. Artificial Environments}

The U.S. government has sponsored underwater, high altitude, and space research for a number of years and has made great progress in identifying many of the problems associated with having humans work in these environments. However, increased utilization of space with the Space Shuttle and the forthcoming Space Station, and increased use of undersea resources by both government agencies and commercial interests emphasize the importance of alleviating problems associated with work there.

Both economic considerations and basic scientific motivations point out the need to increase research funding on the effects of, and methods of dealing with, artificial environments on humans. Working in such environments is generally quite costly because of transportation and environmental control expenses. As a result, maximum productivity is economically very important, and this requires people working with maximum comfort, with the best tools, and with minimum degradation of performance because of illness or injury. Individual safety also has similar requirements. Artificial environments in general, and weightlessness in space flight in particular, also afford unique scientific opportunities to study human physiology without the complicating effects of gravity.

Research in certain areas of aerospace and undersea physiology has been conducted over a number of years by the Air Force, by the Navy, and in recent years, by NASA. However, other research areas will become more important as commercial, scientific, and military exploitation of the space and undersea environments increase in coming years. The following are several key areas in which there is reasonable consensus that increased funding is needed.

1) Physiological changes caused by weightlessness (space) or high pressure (undersea):

- vestibular changes resulting in most of the effects of the Space Adaptation Syndrome (nausea, etc.);

- cardiovascular changes (fluid shifts, stroke volume changes, blood volume loss, etc.);

- electrolyte, bone, and mineral metabolism changes;

- experiments involving increased understanding of functioning of these physiological systems would help to counteract deleterious effects of weightlessness, and also would help ground-based treatment of patients with such disorders as those associated with the neurological/vestibular and cardiovascular systems.

2) Development of improved, reliable, small, easy-touse tools and facilities for remote evaluation, stabilization, and treatment of medical emergencies:

- emphasis should be on easy operation of a miniature medical facility, on an occasional basis, by minimally trained personnel;

- available personnel should have adequate tools to identify and properly treat injuries or other unpredictable medical events, until affected persons can be evacuated;

- artificial intelligence systems may be useful in assisting the nonexpert user in the proper tests and procedures to follow;

- development of such tools would also have numerous spinoffs involving ground-based medical care at remote locations, away from complete medical facilities.

3) Development of improved remote manipulator systems for doing work in hazardous and artificial environments:

- more dexterous manipulators, with better tactile and other feedback to the operator, are needed for performing a large variety of tasks in vacuum or high-pressure (undersea) environments;

- more complex assembly, repair, and inspection tasks would be possible if better manipulators were available, without requiring special suits and long preparation times (examples: assembly of satellites in space by an astronaut in an adjacent pressurized module rather than in a spacesuit; repair of undersea drilling platform components by someone in a small submarine rather than by a diver);

- likely spinoffs from such development work are in industrial robotics, prosthetic devices, and hazardous materials handling.

The above list is not comprehensive, but summarizes several specific areas of key importance. These areas are perhaps more critical than some others because lack of progress in any of them will significantly impede efforts being made for the full utilization of the space and undersea environments. Conversely, progress in these areas will greatly expedite the expanded use of these environments, and will also provide tangible benefits to other areas of national importance. 


\section{CONCLUSIONS}

In addressing the research agenda of biomedical engineering, we have used selected applications within this rather broad field as the basis through which to identify not only issues specific to each, but through these, to identify the more generic issues common to and broadly applicable to much of the field. We note the need for greater research emphasis in the following.

- Improvement of Real-Time Algorithms for Processing Various Biological Signals: While computer engineering and hardware design have seen overwhelming advances over the past decade, computer science and software design have progressed very little. The concepts of artificial intelligence should be refined and integrated into biomedical instruments. There is no medical instrument system now that deserves the frequently used name-expert system. The applications include robotics in rehabilitation medicine, processing schemes for sensor arrays, imaging, patient/provider interactive systems, and cardiac interventional systems. Such research requires the need for real-time algorithm development and expert systems having the ability to monitor sensed information, evaluate that input against a database and decision criteria (with selflearning potential), and initiate an active response.

- Development of Miniature Artificial Sensors and Actuators for Closed-Loop Control of Devices and Drug Delivery: Currently, these are perhaps the weakest link in the development of substitute life functions. Such sensors must go far beyond physical parameter measurements (for which much work has already been done but is not yet resolved) to sensors for body chemistry (in vivo, in vitro, and noninvasive) and chemical processes.

- Understanding the Body's Natural Sensors and How to Interface with Them: These are the critical components to the natural control of numerous, important artificial devices.

- Quantitative Descriptions of Spatially and Temporally Organized Mechanisms of Bioelectric Phenomena of Tissue: While specifically identified within the context of cardiac tissue, many of the questions to be answered are equally applicable to other organ systems: understanding the control strategies of the sensory and motor systems, the manner of activation and response of the body's natural sensors, and nervous system processing.

- Energy Interaction with Biologic Tissues: In particular, we note laser and ultrasonic energy for cardiac care intervention techniques and the consequences of impact trauma. The fundamental questions of this energy/tissue interaction arise equally with respect to imaging modalities and hyperthermia although the specific research approaches and application objectives vary.

The route through which most of the research in biomedical engineering to date has been fruitfully accomplished has been primarily within the academic research community. In recent years, as commercial potential is realized, industrial research in selected cases has been noted in bringing the basic work beyond the proof-of-concept stage to product development. For the most part, the fundamental work is, however, carried out within the academic setting through government sponsorship, private philanthropy, and limited industrial cosponsorships. Despite efforts to increase industrial sponsorship of such academic research, little additional support (on a percentage of total research dollars) is anticipated. The characteristics of such sponsorships typically demand a relatively quick product development route and return to the corporation for the funds invested. Thus, only when the "proofof-concept" stage has been successfully completed and market potential deemed adequate can the industrial sector afford to invest in such R\&D. It is, therefore, anticipated that this research will continue to depend very heavily on federal agency support and direction for fundamental research and development to the "proof-of-concept" stage. This is even more acute in the realm of products for the handicapped where perhaps a concept of "orphan devices" should be established in the manner of "orphan drug" support.

As increased emphasis by both government funding sources and the biomedical engineering research community is placed on the applications of their work, confusion heightens as to the appropriate funding sources. For the biomedical engineering research community, the NIH and NSF are typical research funding routes. However, funding pressures push the NIH in the direction of supporting direct clinically applicable biomedical research, with a resultant decrease in the fundamental research of the engineering and physical science phenomena that may offer better hope of physiologic explanation, product development, and ultimately patient care. Yet, while the mission of NSF appears to be broadening to include application as well as very basic science, within the biomedical engineering community the application point leads ultimately to clinical care. To the NSF, this translates to a project that should be supportable by the NIH. Thus, the biomedical engineering research community finds itself in serious difficulty with the potencial for falling between the perceived missions of these two agencies without either assuming responsibility. We urge an examination of this and the recognition by (and increased funding for) both the NIH and the NSF to support the fundamental work of the engineering and physical sciences underlying biomedical engineering development to the "proof-of-concept" stage. This should be true at the NSF even if the developments may have future (in the long term) clinical applicability and should be true for the NIH (which must look to the long as well as the short term) because of potential clinical applicability. The problem posed for a multidisciplinary program encompassing the marriage of engineering and physical sciences, on the one hand, with the life and clinical sciences, on the other, is not totally unexpected in view of the disciplinary character of the structure of this field of government support. It does, however, become 
more acute as both agencies are pressed to identify more narrowly their application foci and does require attention lest this field quickly find itself without an advocate within the federal research structure.

\section{ACKNOWLEDGMENT}

The authors are grateful for the technical assistance of and suggestions from the following individuals: D. Childress, Rehabilitation Institute of Chicago; J. Eulenberg, Michigan State University; D. Jaffee, Palo Alto VA Medical Center; R. Ideker, Duke University; D. McNeal, Rancho Los Amigos Hospital; J. Melvin, University of Michigan at Ann Arbor; V. Nickel, Sharp Rehabilitation Center; B. Romich, Prentke-Romich Company; G. Trahey, Duke University; J. Trimble, VAMC Hines; and M. Wolbarsht, Duke University.

\section{REFERENCES}

[1] G. Bugliarello, "Health care costs: Technology to the rescue?" IEEE Spectrum, vol. 21, no. 6, pp. 97-100, 1984.

[2] N. V. Thakor, "From Houlter monitors to automatic defibrillators: Developments in ambulatory arrhythmia monitoring," IEEE Trans. Biomed. Eng., vol. BME-31, pp. 770-778, Dec. 1984.

[3] F. Pannizzo, "Biomedical engineerings' greatest contribution," IEEE Eng. Med. Biol. Mag., vol. 3, no. 2, p. 9, 1984.

[4] Y. Rudy, Guest Ed. (Special Issue) Ann. Biomed. Eng., vol. 11, nos. 3/4, pp. 147-262, 1983.

[5] T. C. Pilkington and R. Plonsey, Engineering Contributions to Biophysical Electrocardiography. New York: IEEE Press, 1982.

[6] G. Lee, R. Ikeda, and I. Herman, "The qualitative effects of laser irradiation on human arteriosclerotic heart disease," Amer. Heart J., vol. 105 , no. 6 , pp. 885-889, 1983.

[7] W. S. Andrus, "Laser angioplasty combats heart disease: New surgical laser procedures await improvements in fiber optics," Laser Appl., pp. 97-100, Feb. 1985.

[8] M. Blois, Information in Medicine: The Nature of Medical Descriptions. Berkeley, CA: University of California Press, 1984.

[9] P. Szolovits, Artificial Intelligence in Medicine. Boulder, CO: Westview, 1982.

[10] W. J. Clancey and E. H. Shortliffe, Readings in Medical Artificial Intelligence: The First Decade. Reading, MA: Addison-Wesley, 1984.

[11] D. A. Fenderson, "Computers to aid the handicapped," Computer, vol. 17, pp. 301-303, 1984.

[12] R. L. Jeffcoat, K. M. Captain, and D. M. Hudson, "Machine vision in industry," Med. Dev. Diagnos. Ind., vol. 5, pp. 93, 1983.

[13] G. Silverman, "Automation in the biomedical laboratory," IEEE Trans. Biomed. Eng., vol. BME-31, pp. 748-752, Dec. 1984.

[14] R. R. Schreiber, "Robotics unlimited: Reaching beyond the factory," Robot. Today, vol. 6, pp. 43-47, 1984.

[15] J. Horgan, "Technology '84: Medical electronics," IEEE Spectrum, pp. 90-93, Jan. 1984.

[16] "Technology and handicapped people," Rep. from the Office of Technology Assessment, Washington, DC, 214 p., May 1982.

[17] "Technology and handicapped people," a joint hearing before the Subcommittee on Science, Research and Technology of the Committee on Science and Technology, U.S. House of Representatives and the Committee on Labor and Human Resources, U.S. Senate, 97th Congr., 2nd Session, Sept. 29, 1982, no. 163, 263 p.

[18] National Center for Health Statistics, Advance Rep. final mortality statistics, 1980, Monthly Vital Statistics Rep., Suppl. 1, Aug. 1983, vol. 32 , no. 4 .

[19] N. S. Hartunian, C. N. Smart, and M. S. Thompson, The Incidence and Economic Costs of Major Health Impairments: A Comparative Analysis of Cancer, Motor Vehicle Injuries, Coronary Heart Disease and Stroke. Lexington, MA: Lexington Books, 1981.

[20] The Biomechanics of Trauma, A. M. Nahum and J. Melvin, Eds. Norwalk, CT: Appleton, 1985.

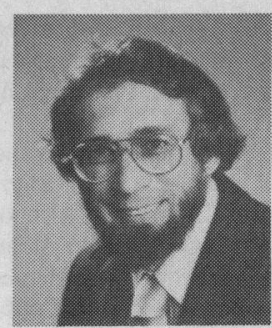

Alfred R. Potvin (S'68-M'71-SM'78) received the B.S. degree in electrical engineering from Worcester Polytechnic Institute, Worcester, MA, the M.S. degree and the Engineer degree in electrical engineering from Stanford University, Stanford, CA, and the M.S. degree in psychology and the M.S. and Ph.D. degrees in bioengineering from the University of Michigan, Ann Arbor.

$\mathrm{He}$ is currently the Director of the Medical Instrument Systems Research Division at Eli Lilly and Company. From 1966 to 1984 he was a Professor at the University of Texas at Arlington and served as Founding Chairman of Biomedical Engineering and Director of the Rehabilitation Engineering Research Center. He is the author of two books and over 250 publications. He was the Chairman of the ASEE-Biomedical Engineering Division, a member of the AEMB Council, and Chairman of the Education and Public Affairs Committee of BMES. He has served on the VA Rehabilitation Research and Development Merit Review Board, the FDA Physical Medicine Section of the Surgical and Rehabilitation Devices Panel, the Board of Directors for RMBS, and the Advisory Board of the Southern Biomedical Engineering Conference. He currently serves on the Advisory Board for NSF's Division of Fundamental Research for Emerging and Critical Engineering Systems.

Dr. Potvin has been a Conference Chairman and President of the IEEE Engineering in Medicine and Biology Society.

William G. Crosier (S'75-M'76) received the B.S. degree in electrical engineering from the University of Texas at Arlington in 1973, and the M.S. degree in biomedical engineering jointly from the University of Texas at Arlington and the University of Texas Health Science Center at Dallas in 1975.

From 1975 to the present he has worked for Technology, Inc., Houston, TX. There he has worked on instrumentation and software development for the NASA/JSC Life Sciences Research Laboratories. He began there as the Staff Biomedical Engineer for the Cardiovascular Research Laboratory, and has since worked primarily in the Neurophysiology Laboratory on vestibular research projects. He is now the Senior Engineer for the Biomedical Research Laboratories. His major interests are biomedical instrumentation, microcomputer hardware and software, and human factors.

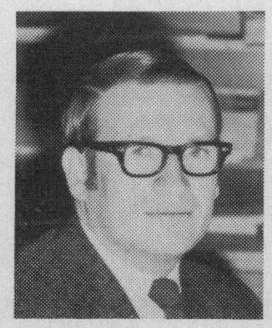

Eli Fromm (S'60-M'65-SM'77-F'85) received the B.S.E.E. and M.S. degrees from Drexel University, Philadelphia, PA, in 1962 and 1964, respectively, and the Ph.D. degree from the Jefferson Medical College of Thomas Jefferson University, Philadelphia, PA, in 1967.

In 1962 he joined the Life Support Systems Group, General Electric Company and, in 1963, the Applied Physics Laboratory, E.I. DuPont. After graduate studies as a National Institutes of Health Special Fellow, he joined the Faculty of Drexel University, Philadelphia, PA, with positions in the Biomedical Engineering and Science Institute, and the Department of Biological Sciences. At present he is a Professor in the Department of Electrical and Computer Engineering. In 1980 and 1981 he served the U.S. Congress, having been selected as a Congressional Science and Engineering Fellow. Currently he also serves at the National Science Foundation directing the bioengineering program. His research interests center on bioinstrumentation, sensors, biotelemetry and their applications to physiologic evaluation, and health care systems.

Dr. Fromm is a member of Tau Beta Pi, Eta Kappa Nu, and Sigma Xi. He twice served as President of the IEEE Engineering in Medicine and Biology Society (EMBS), is currently a member of the IEEE Board of Directors, the Technical Activities Board, the United States Activities Board, and the Educational Activities Board. 


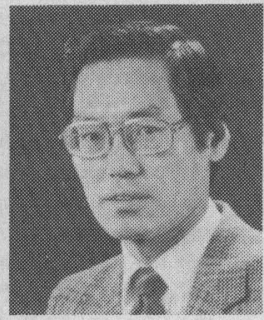

James C. Lin (S'65-S'67-M'71-SM'77) received the B.S., M.S., and Ph.D. degrees in electrical engineering from the University of Washington, Seattle.

Presently he is the Director of the Robotics and Automation Laboratory in the College of Engineering at the University of Illinois at Chicago, where he also serves as a Professor and the Head of the Department of Bioengineering. He has published over 100 papers and two books. One publication received an IEEE TRANSACTIONS Prize Paper Award (1975). He has been a Scientific Consultant to numerous private organizations as well as state and federal agencies.

Dr. Lin is a member of four editorial boards. He has served on the IEEE Committee on Man and Radiation (COMAR), American National Standards Institute (ANSI) and Subcommittee C95.4, USNC/URSI of the National Academy of Sciences. He has been a member of the NIH Diagnostic Radiology Study Section and Special Study Sections for SBIR. He also served on the Governor's Task Force to Review Project Seafarer (Michigan, 1976) and on the NSF ECS Division Panel for the Presidential Young Investigators Award (1984). He has been a board member of two technical societies and is a senior member of Robotics International, the Biomedical Engineering Society and the IEEE. He presently serves as the Chairman of the EMBS Committee on Biomedical Robotics, and as Program Chairman for the 1985 EMBS Annual Conference in Chicago.

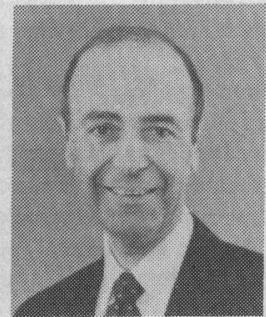

Michael R. Neuman (M'62) received the B.S., M.S., and Ph.D. degrees in electrical engineering at Case Institute, Cleveland, $\mathrm{OH}, 1961,1963$, and 1966, respectively. He received the M.D. degree from the Case Western Reserve University School of Medicine in 1974 while on the Faculty of the Department of Electrical Engineering.

In 1980 he was a guest Professor in the Department of Obstetrics and Gynecology of the University of Zurich, Switzerland. He currently serves as an Associate Professor of Biomedical Engineering in the Department of Reproductive Biology at Case Western Reserve University.

Dr. Neuman is a member of various professional organizations including the Editorial Board of the IEEE TRANSACTIONS ON BIOMEDICAL ENGINEERING and the Journal of Clinical Engineering. He is also currently the President of the International Society of Biotelemetry.

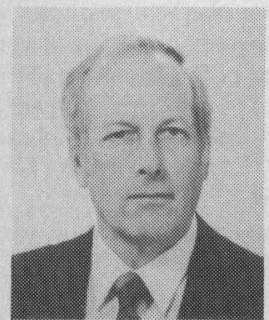

Theo C. Pilkington (S'54-M'59-SM'84-F'85) received the B.S.E.E. degree from North Carolina State, Raleigh, in 1958, the M.S.E.E. degree in 1960 , and the $\mathrm{Ph} . \mathrm{D}$. degree in 1963, both from Duke University, Durham, NC.

$\mathrm{He}$ is a Professor of Biomedical Engineering and Electrical Engineering at Duke University where he has taught and done research since 1961 . From 1967 to 1979 he was the Chairman of Biomedical Engineering at Duke. He has done postdoctoral study at Duke and M.I.T. and has been a Fellow-by-Courtesy at Johns Hopkins University, Baltimore, MD. $\mathrm{He}$ has served as the Editor of the CRC Critical Reviews in Bioengineering (1976-1979).

Dr. Pilkington was the Editor of the IEEE TRANSACTIONS ON BIOMEDICAL ENGINEERING (1979-1984), and presently serves on the Editorial Board of the PROCEedings of tHE IEEE.

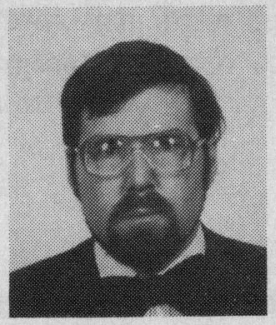

Charles J. Robinson (S'68-M'71-S'75-M'79SM'82) is the Associate Director of the Rehabilitation R\&D Center at the VA Hospital in Hines, IL, and has had faculty appointments in the Department of Physiology at Loyola University School of Medicine and in the Bioengineering Program at the University of Illinois, Chicago. His major research areas include: electrical stimulation of paralyzed muscle; studies of bladder dysfunction following spinal injury; animal models of stroke; and the neurophysiology of pain and touch. He previosly was employed as a Member of the Technical Staff at Bell Laboratories, Columbus, $\mathrm{OH}$, where he studied the surface physics of sealed contacts. He has authored over 50 papers and abstracts describing his work.

Dr. Robinson is a member of a number of international organizations, and is a Registered Professional Engineer. He has served on the Program Committee for the 1984 IEEE Engineering in Medicine and Biology Society meeting and was Vice President of his student chapter. He is on the Editorial Board of the IEEE ENGINEERING IN MEDiCine AND BIology MAGAZINE and is the Chairman of the EMBS TIC on Rehabilitation. He organized the very successful informal gathering of rehabilitation engineers at the 1984 EMBS annual meeting.

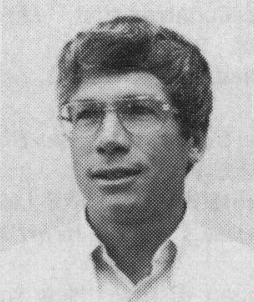

Lawrence W. Schneider received the B.S.E. degree in mechanical engineering from the University of Michigan in 1965 and the M.S.E. degree in mechanical engineering in 1966. After serving two years in the U.S. Navy he returned to graduate school at the University of Michigan where he conducted his dissertation research work in vestibular neurophysiology at the Kresge Hearing Research Institute and obtained the Ph.D. degree in biomedical engineering in 1973.

For the past seven years he has been on the re search Staff of the University of Michigan Transportation Research Institute (UMTRI-formerly HSRI) where he has conducted research in a number of areas including anthropometry of children related to product safety design, biomechanical properties of the human head and neck related to dynamic impact response, occupant protection for the severely disabled, and ergonomic studies of driver controls.

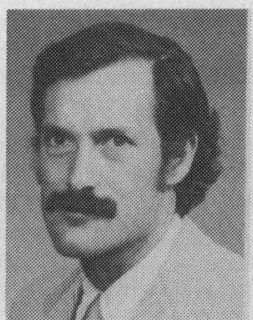

John W. Strohbehn (S'57-M'64-SM'81) received the B.S., M.S., and Ph.D. degrees in electrical engineering from Stanford University, Stanford, CA, in 1958, 1959, and 1964, respectively.

He joined the Faculty at the Thayer School of Engineering, Dartmouth College, Hanover, NH, in 1963, where he presently holds a position as Professor of Engineering. His research efforts have been in the field of radiophysics, including microwave and optical propagation through the atmosphere, and in biomedical engineering, including image processing, tomography, and the use of heat in the cure and control of cancer.

Dr. Strohbehn is a Fellow of the Optical Society of America, a member of the American Association for the Advancement of Science and URSI Commission II. He was a National Academy Exchange Scientist to the Soviet Union in 1964, an Associate Editor of the IEEE TRANSACTIONS ON ANtenNas AND Propagation from 1969 to 1971 , and is presently Associate Editor of the IEEE TRANSACTIONS ON BIOMEDICAL ENGINEERING. He was a Visiting Research Scientist at Stanford University Medical School in 19811982. 


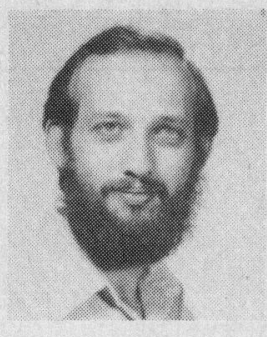

Peter Szolovits received the B.S. degree in physics in 1970, and the Ph.D. degree in information science in 1974, both from the California Institute of Technology, Pasadena.

He has been on the Massachusetts Institute of Technology Faculty since 1974, where he is now an Associate Professor of Computer Science and Engineering. He is the Head of the Clinical Decision Making Group in the M.I.T. Laboratory for Computer Science, and is in charge of the Computer Science graduate program within the Department of Electrical Engineering and Computer Science. His research currently focuses on the application of artificial intelligence techniques to medical diagnostic and therapeutic decision making, and on the development of new methods of representing knowledge of the world in the computer in forms that support their use for problem solving and explanation. He has also done some work on problems of natural language understanding and the use of "expert systems" methods for building practical commercial programs.

Dr. Szolovits is a Fellow of the American College of Medical Informatics and a member of the American Association for Artificial Intelligence, the Association for Computing Machinery, and Sigma Xi.

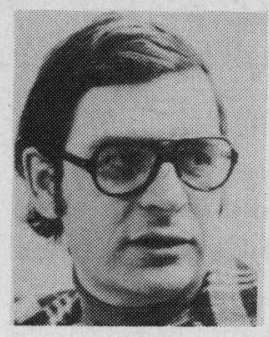

Willis J. Tompkins (S'61-M'66-SM'77) received the B.S. and M.S. degrees in electrical engineering from the University of Maine, Orono, in 1963 and 1965 , respectively. He received the Ph.D. degree in biomedical electronic engineering from the University of Pennsylvania, Philadelphia, in 1973.

From 1965 to 1968 he was an Electrical Engineer at Sanders Associates, Inc., Nashua, NH, where he worked on research and development of data storage systems. He was employed from 1973 to 1974 at the Hospital of the University of Pennsylvania as a Biomedical Engineer. Since 1974, he has been on the Faculty of the University of Wisconsin-Madison. Currently he is a Professor of Electrical and Computer Engineering, and teaches undergraduate, graduate, and short courses on the topic of computers in medicine. His research interests include on-line biomedical computing and microcomputer-based instrumentation applications. He is a Co-editor, with J. G. Webster, of the textbook Design of Microcomputer-Based Medical Instrumentation (Englewood Cliffs, NJ: Prentice-Hall, 1981) and Co-editor, with J. G. Webster, A. M. Cook, and G. C. Vanderheiden, of the textbook Electronic Devices for Rehabilitation (New York: Wiley, 1985).

Dr. Tompkins is a member of the IEEE Engineering in Medicine and Biology Society (currently Region IV Administrative Committee Representative and Vice President for Technical Activities), the IEEE Computer Society, the Association for the Advancement of Medical Instrumentation, and the Biomedical Engineering Society. He is a Registered Professional Engineer in Wisconsin. 